\title{
Entropy and Global Markov Properties
}

\author{
S. Goldstein ${ }^{1,2}$, R. Kuik ${ }^{1,2,3}$ and A. G. Schlijper ${ }^{4}$ \\ 1 Department of Mathematics, Rutgers University, New Brunswick, New Jersey 08903, USA \\ 2 Research supported by NSF Grants DMS 85-12505 and SMR 86-12369 \\ 3 Permanent address: EUR, Faculteit Bedrijfskunde, P.O. Box 1738, NL-3000 DR Rotterdam, The \\ Netherlands \\ ${ }^{4}$ Koninklijke/Shell Exploratie en Produktie Laboratorium, P.O. Box 60, NL-2280 AB Rijswijk, The \\ Netherlands
}

\begin{abstract}
We extend, refine and give simple proofs of some recent results on the validity of global Markov properties for classical spin systems. One of the new results is that there is a global Markov property that is satisfied by equilibrium states in general. The proof of this establishes formulas for the entropy and free energy that show that these quantities are, for $d$-dimensional systems, given in terms of $(d-1)$-dimensional systems. Furthermore, we show that global Markov properties imply the absence of some types of symmetry breaking.
\end{abstract}

\section{Introduction}

Consider a classical spin system on $\mathbb{Z}^{d}$ with a translation invariant interactionround-faces potential $\Phi=\left\{\Phi_{X}\right\}_{\substack{X \subset \mathbb{Z}^{d} \\ X \subset \text { a unit } d \text {-cube }}}$ on the configuration space $\Omega \equiv \Omega_{0}^{\mathbb{Z}^{d}}$,

where $\Omega_{0}$ is a finite set and $\Phi_{X}: \Omega_{X} \equiv \Omega_{0}^{X} \rightarrow \mathbb{R}$ are real functions. A state $\mu$ is a Gibbs state for the potential $\Phi$ if it satisfies the DLR equations,

$$
\mu_{\Lambda}\left(\sigma_{\Lambda} \mid \sigma_{\Lambda^{c}}\right)=\frac{1}{Z_{\sigma_{\Lambda^{c}}}} \exp \left[-H_{\Lambda}\left(\sigma_{\Lambda}\right)-W_{\Lambda}\left(\sigma_{\Lambda}, \sigma_{\Lambda^{c}}\right)\right]
$$

for each finite $\Lambda \subset \mathbb{Z}^{d}$, where $\Lambda^{c}$ is the complement of $\Lambda, W_{\Lambda}$ is the function on $\Omega_{\Lambda} \times \Omega_{\Lambda^{c}}$ defined by

$$
W_{\Lambda}\left(\sigma_{\Lambda}, \sigma_{\Lambda^{c}}\right)=\sum_{\substack{X: X \cap \Lambda \neq \phi \\ X \cap \Lambda^{c} \neq \phi}} \Phi_{X}\left(\sigma_{X}\right)
$$

and where $Z_{\sigma_{\Lambda^{c}}}$ is determined by the normalisation

$$
\sum_{\sigma_{\Lambda} \in \Omega_{\Lambda}} \mu\left(\sigma_{\Lambda} \mid \sigma_{\Lambda^{c}}\right)=1
$$


The DLR equations for Gibbs states immediately imply

$$
E\left(f_{\Lambda} \mid \Lambda^{c}\right)=E\left(f_{\Lambda} \mid \partial \Lambda\right)
$$

for every finite $\Lambda \subset \mathbb{Z}^{d}$, where

$$
\partial \Lambda=\left\{x \in \mathbb{Z}^{d} \inf _{y \in \Lambda} \sup _{i=1, \ldots, d}\left|x_{i}-y_{i}\right|=1\right\}
$$

and $E\left(. \mid \Lambda^{\prime}\right)$ is the conditional expectation given the spins in $\Lambda^{\prime} \subset \mathbb{Z}^{d}$ and $f_{\Lambda}$ is a function that depends only on spins in $\Lambda$.

Property (1) expresses that Gibbs states satisfy the Markov property for every finite $\Lambda \subset \mathbb{Z}^{d}$. If (1) holds for a specific infinite $\Lambda$, then we say that the state satisfies the global Markov property for $\Lambda$.

The state is said to satisfy the global Markov property if (1) holds for any, possibly infinite, $\Lambda$. The global Markov property has been established for Gibbs states when the Dobrushin uniqueness criterion applies and also for the \pm states for attractive potentials $[1,2,3]$.

Since Gibbs states have local Markov properties, one is naturally inclined to establish global Markov properties by "continuity" arguments: letting (finite) $\Lambda, s$ grow to an infinite volume while keeping control of (1). However, in general, this turns out to be a subtle procedure and indeed it has to to be subtle since several examples of the failure of global Markov properties exist [2, 4]. In [4] Israel shows that the global Markov property may fail even for extremal Gibbs states which are translation-invariant.

On the other hand, it is shown in [5] that for equilibrium states, i.e., translation-invariant Gibbs states, under conditions of much greater generality than those in previous work, one has the global Markov property for $\Lambda_{+} \equiv\left\{x_{1}>0\right\}$. Note that this Markov property is naturally equivalent to these Gibbs states as defining stationary Markov chains on $\Omega_{\mathbb{Z}^{d-1}}$. The argument for the above Markov property is not based directly on local Markov properties but uses the variational principle which states that equilibrium states minimise the free energy. This argument goes roughly as follows:

Let $\mu$ be an equilibrium state for $\Phi$ and let $\mu_{2}$ be its projection onto (the configurations on) the double layer $\left\{x_{1}=0,1\right\}$. Using $\mu_{2}$, one now constructs another translation-invariant state $\bar{\mu}$ on $\Omega$ such that

(1) the projection of $\bar{\mu}$ onto $\left\{x_{1}=0,1\right\}$ equals $\mu_{2}$,

(2) $\bar{\mu}$ has the global Markov property for $\Lambda_{+}$.

In fact $\bar{\mu}$, which is just the Markov chain on $\Omega_{\mathbb{Z}^{d-1}}$ arising from $\mu_{2}$, is the unique translation-invariant state with the properties (1) and (2). Thus the state $\bar{\mu}$ can be thought of as a "Markovization" of $\mu$. The important observation at this point is that the entropy density of $\bar{\mu}, s(\bar{\mu})$, is not smaller than the entropy density of $\mu$, $s(\mu)$, i.e.

$$
s(\bar{\mu}) \geqq s(\mu) .
$$

Since the energy densities of the states $\mu$ and $\bar{\mu}$ are the same, the variational principle now implies that $\bar{\mu}$ is also an equilibrium state. Thus we have an equilibrium state with a global Markov property. So, once (2) has been noted, one immediately has the following result, which is essentially the main result in [5]. 
Theorem 0. Let $\mu$ be an equilibrium state for $\Phi$ and consider Condition $C(\mu)$,

$$
\begin{aligned}
& \text { If } \mu^{\prime} \text { is an equilibrium state for } \Phi \text { that } \\
& \text { has the same projection onto }\left\{x_{1}=0,1\right\} \\
& \text { as } \mu \text {, then } \mu=\mu^{\prime} .
\end{aligned}
$$

If $C(\mu)$ holds then $\mu$ has the global Markov property for $\Lambda_{+}$. Moreover, if $C(\mu)$ fails, then there are equilibrium states with the same projection on $\left\{x_{1}=0,1\right\}$ as $\mu$, for which the global Markov property for $\Lambda_{+}$fails.

The last assertion of the theorem is a consequence of the uniqueness of $\bar{\mu}$.

The crucial entropy inequality (2) was noted in [6]. Below, we present a simple proof of it. We also study a "Markovization" different from that sketched above and proposed in the appendix to [6], to derive, along analogous lines, some further Markov properties which are general in that each equilibrium states satisfies them. As a related result we establish a formula for the entropy density of equilibrium states. One consequence of this formula is that one can formulate a variational principle for $d$-dimensional systems in terms of $(d-1)$-dimensional systems. In the final section we show that Markov properties sometimes allow one to conclude that periodic states are invariant under $\mathbb{Z}_{\mathrm{ev}}^{d}$, where

$$
\mathbb{Z}_{\mathrm{ev}}=\{n \in \mathbb{Z} \mid n \text { is even }\} .
$$

The results and proofs are given for arbitrary dimension $d$ but it may be instructive to have $d=2$ in mind when reading the next sections.

\section{Preliminaries}

In this section we present the variational principle and a formula for the entropy which is needed in the next section.

We begin by introducing some notation. For $\Lambda \subset \mathbb{Z}^{d}$ we denote a configuration on $\Lambda$ by $\sigma_{\Lambda}$, i.e., $\sigma_{\Lambda} \in \Omega_{\Lambda}$. We write $\sigma_{\mathbb{Z}^{d}} \equiv \sigma \in \Omega$. Whenever we refer to two configurations $\sigma_{\Lambda}$ on $\Lambda$ and $\sigma_{\Lambda^{\prime}}$ on $\Lambda^{\prime}$ at the same time it will be understood that these configurations agree on $\Lambda \cap \Lambda^{\prime}$. The set of continuous functions on $\Omega$ which depend only on spins in $\Lambda$ is denoted by $\mathrm{C}_{\Lambda}$. Furthermore, we denote the $\sigma$-algebra generated by the spins in $\Lambda$ by $B_{\Lambda}$.

A state is a probability measure and the set of translation-invariant states on $\boldsymbol{\Omega}$ is denoted by $I$. When $\Lambda \subset \mathbb{Z}^{d}$, a state $v_{\Lambda}$ on $\Omega_{\Lambda}$ will be called (locally) translation invariant if $f, g \in C_{\Lambda}$ and $\mu(f)=\mu(g)$ for all $\mu \in I$ imply that $v_{\Lambda}(f)=v_{\Lambda}(g)$. Here $\mu(f)$ stands for $\int f d \mu$, etc. When $\mu$ is a state on $\Omega$ we denote its projection on $\Omega_{\Lambda}$ by $\mu_{\Lambda}$ and we will sometimes refer to $\mu$ as an extension of $\mu_{\Lambda}$. For $\Lambda$ finite we define the entropy of a state $\mu, S_{\Lambda}(\mu)$, by

$$
S_{\Lambda}(\mu)=-\sum_{\sigma_{\Lambda} \in \Omega_{\Lambda}} \mu_{\Lambda}\left(\sigma_{\Lambda}\right) \ln \mu_{\Lambda}\left(\sigma_{\Lambda}\right)
$$

For $\mu \in I$ the entropy density, $s(\mu)$, is defined by

$$
s(\mu)=\lim _{\Lambda \uparrow \mathbb{Z}^{d}} \frac{1}{|\Lambda|} S_{\Lambda}(\mu),
$$

where the limit is taken in the van Hove sense. The Hamiltonian for a finite volume 
$\Lambda, H_{\Lambda}$, is the function on $\Omega_{\Lambda}$ defined by

$$
H_{\Lambda}\left(\sigma_{\Lambda}\right)=\sum_{X \subset \Lambda} \Phi_{X}\left(\sigma_{X}\right)
$$

and the free energy for the volume $\Lambda, F_{\Phi}(\Lambda)$ is defined by

$$
F_{\Phi}(\Lambda)=-\ln \left(\sum_{\sigma_{\Lambda} \in \Omega_{\Lambda}} \exp \left(-H_{\Lambda}\left(\sigma_{\Lambda}\right)\right)\right)
$$

Here we adopt the convention that the inverse temperature is set equal to 1 . The free energy density, $f_{\Phi}$, is given by

$$
f_{\Phi}=\lim _{\Lambda \uparrow \mathbb{Z}^{d}} \frac{1}{|\Lambda|} F_{\Phi}(\Lambda) .
$$

Define the function $e_{\Phi}$ by

$$
e_{\Phi}(\sigma)=\sum_{X: o \in X}^{*} \Phi_{X}\left(\sigma_{X}\right)
$$

where $\sum^{*}$ means that the sum runs over those subsets that have $\underline{o}=(0, \ldots, 0)$ as their last element in the lexicographic order of $\mathbb{Z}^{d}$. The expectation value $\mu\left(e_{\Phi}\right)$ of $e_{\Phi}$ in a translation-invariant state $\mu$ is the energy density of this state.

We denote the conditional measure for a state $\mu$ given the configuration $\sigma_{\Lambda} \in \Omega_{\Lambda}$ by $\mu\left(. \mid \sigma_{\Lambda}\right)$ and the projection of this measure on $\Lambda^{\prime}$ by $\mu_{\Lambda^{\prime}}\left(. \mid \sigma_{\Lambda}\right)$. The following theorem shows that translation-invariant Gibbs states are characterised by a variational principle.

Theorem 1 (Variational Principle). For any $\mu \in I$

$$
e(\mu)-s(\mu) \geqq f_{\Phi}
$$

and equality holds if and only if the state $\mu$ is a Gibbs state for $\Phi$.

For a proof of this theorem see e.g. [9].

For $\Lambda$ finite we define the conditional entropy given $\Lambda^{\prime} \subset \mathbb{Z}^{d}$ of a state $\mu$ by

$$
S_{\Lambda \mid \Lambda^{\prime}}(\mu)=\int \mu_{\Lambda^{\prime}}\left(d \sigma_{\Lambda^{\prime}}\right) S_{\Lambda}\left(\mu\left(. \mid \sigma_{\Lambda^{\prime}}\right)\right) .
$$

$\left[S_{\Lambda \mid \phi}(\mu)=S_{\Lambda}(\mu).\right]$ Note that for $\Lambda$ and $\Lambda^{\prime}$ both finite

$$
S_{\Lambda \mid \Lambda^{\prime}}(\mu)=S_{\Lambda \cup \Lambda^{\prime}}(\mu)-S_{\Lambda^{\prime}}(\mu) .
$$

Lemma 1, see e.g. [9]. Conditional entropy has the monotonicity property

$$
\Lambda^{\prime \prime} \subset \Lambda^{\prime} \Rightarrow S_{\Lambda \mid \Lambda^{\prime \prime}}(\mu) \geqq S_{\Lambda \mid \Lambda^{\prime}}(\mu) \text {. }
$$

Moreover, equality of these conditional entropies holds if and only if

$$
\mu_{\Lambda}\left(. \mid \sigma_{\Lambda^{\prime}}\right)=\mu_{\Lambda}\left(. \mid \sigma_{\Lambda^{\prime \prime}}\right)(\mu \text { almost surely })
$$

Proof. By Jensen's inequality

$$
-\mu_{\Lambda}\left(\sigma_{\Lambda} \mid \sigma_{\Lambda^{\prime \prime}}\right) \ln \mu_{\Lambda}\left(\sigma_{\Lambda} \mid \sigma_{\Lambda^{\prime \prime}}\right) \geqq-\int \mu_{\Lambda^{\prime}}\left(d \sigma_{\Lambda^{\prime}} \mid \sigma_{\Lambda^{\prime \prime}}\right)\left(\mu_{\Lambda}\left(\sigma_{\Lambda} \mid \sigma_{\Lambda^{\prime}}\right) \ln \mu_{\Lambda}\left(\sigma_{\Lambda} \mid \sigma_{\Lambda^{\prime}}\right)\right) .
$$

Summing this inequality over $\sigma_{\Lambda} \in \Omega_{\Lambda}$ and integrating it with respect to $\mu_{\Lambda^{\prime \prime}}\left(d \sigma_{\Lambda^{\prime \prime}}\right)$ yields the inequality of the lemma. If the inequality of the lemma is an equality, 
then the above inequality $(*)$ has to be an equality too ( $\mu$ almost surely). Hence $\mu_{\Lambda}\left(\sigma_{\Lambda} \mid \sigma_{\Lambda^{\prime}}\right)$ is constant (almost surely) with respect to $\mu_{\Lambda^{\prime}}\left(d \sigma_{\Lambda^{\prime}} \mid \sigma_{\Lambda^{\prime \prime}}\right)$ and therefore equal to its expectation value for this measure which is $\mu_{\Lambda}\left(\sigma_{\Lambda} \mid \sigma_{\Lambda^{\prime \prime}}\right)$.

We order the lattice $\mathbb{Z}^{d}$ lexicographically and $x<y$ for $x, y \in \mathbb{Z}^{d}$ will mean $\left(x_{1}<y_{1}\right)$ or $\left(x_{1}=y_{1}\right.$ and $\left.x_{2}<y_{2}\right)$ or $\cdots$ or $\left(x_{1}=y_{1}\right.$ and $\cdots$ and $x_{d-2}=y_{d-2}$ and $\left.x_{d-1}<y_{d-1}\right)$ or $\left(x_{1}=y_{1}\right.$ and $\cdots x_{d-1}=y_{d-1}$ and $\left.x_{d}<y_{d}\right)$.

The following well-known proposition, see e.g. [7], states that the entropy density of a translation-invariant state on $\Omega$ can be expressed as a conditional entropy. For completeness, we provide a proof.

Theorem 2. Consider $\mu \in I$. Then

$$
s(\mu)=S_{\{0\} \mid\{x<0\}}(\mu)
$$

with $\underline{\underline{o}}=(0, \ldots, 0) \in \mathbb{Z}^{d}$ and $\{x<\underline{o}\}$ a notational abbreviation for $\left\{x \in \mathbb{Z}^{d} \mid x<\underline{o}\right\}$.

Proof. Set $V_{n}=\left\{\left|x_{i}\right| \leqq n ; i=1, \ldots, d\right\}$ for $n \in \mathbb{N}$ and set $V_{n}(y)=V_{n} \cap\{x<y\}$. Define

$$
\bar{s}=S_{\{0\}\{x<o\}}(\mu) .
$$

Since $\mu_{\{\underline{\{0\}}}\left(. \mid \sigma_{V_{n}(0)}\right) \rightarrow \mu_{\{0\}}\left(. \mid \sigma_{\{x<0\}}\right)$ for $n \rightarrow \infty$, by monotonicity of the conditional entropy, $\bar{s}=\inf _{n} S_{\{0\} \mid V_{n}(0)}(\mu)$ and, moreover, since $\mu$ is translation-invariant,

$$
\bar{s} \leqq S_{\{y\} \mid V_{n}(y)}(\mu) .
$$

Now

$$
S_{V_{n}}(\mu)=\sum_{y \in V_{n}} S_{\{y\} \mid V_{n}(y)}(\mu) .
$$

Therefore

$$
s(\mu)=\lim _{n \rightarrow \infty} \frac{1}{\left|V_{n}\right|} S_{V_{n}}(\mu) \geqq \bar{s} .
$$

On the other hand take $N \in \mathbb{N}$ and consider

$$
W_{n}=\left\{y \in V_{n} \mid \exists \text { translation } \tau \text { such that } \tau V_{n}(y) \supset V_{N}(\underline{o})\right\} .
$$

Now note that $\lim _{n \rightarrow \infty}\left|W_{n}\right| /\left|V_{n}\right|=1$ and $S_{\{y\} \mid V_{n}(y)}(\mu) \leqq S_{\{0\} \mid V_{N}(0)}(\mu)$ for $y \in W_{n}$. Thus $s(\mu)=$ $\lim _{n \rightarrow \infty}\left(1 /\left|V_{n}\right|\right) S_{V_{n}}(\mu) \leqq S_{\{0\} \mid V_{N}(0)}(\mu)$. Hence $s(\mu) \leqq \bar{s}$, and we conclude $s(\mu)=\bar{s}$.

\section{Markov Properties and Entropy}

Theorem 2 in the previous section states that entropy density equals a conditional entropy. Conditional entropy gives information on conditional measures and thus can give information on Markov properties. Therefore, one may try to establish Markov properties by using entropy considerations. The following lemma shows how entropy considerations and the variational principle can be put in tandem to establish Markov properties of equilibrium states. 
Lemma 2. Let $\mu$ be a translation-invariant Gibbs state for $\Phi$ and let $v$ be a translation-invariant state with the same energy density as $\mu$, i.e.

$$
v\left(e_{\Phi}\right)=\mu\left(e_{\Phi}\right)
$$

Furthermore, let $\Lambda \subset\{x<\underline{o}\}$ be such that

(a) $v_{\Lambda \cup\{0\}}=\mu_{\Lambda \cup\{0\}}$,

(b) $v_{\{o\}}\left(. \mid \sigma_{\{x<o\}}\right)=v_{\{o\}}\left(. \mid \sigma_{\Lambda}\right) \quad(v$ almost surely).

Then

(A) $v$ is an equilibrium state for $\Phi$,

(B) $\mu_{\{\underline{o}\}}\left(. \mid \sigma_{\{x<o\}}\right)=\mu_{\{\underline{0}\}}\left(. \mid \sigma_{\Lambda}\right) \quad$ ( $\mu$ almost surely).

Proof. By the variational principle

$$
v\left(e_{\Phi}\right)-s(v) \geqq \mu\left(e_{\Phi}\right)-s(\mu)=f_{\Phi} .
$$

Therefore $s(v) \leqq s(\mu)$. Hence

$$
s(v) \leqq s(\mu)=S_{\{0\} \mid\{x<0\}}(\mu) \leqq S_{\{\underline{o}\} \mid \Lambda}(\mu)=S_{\{\underline{o}\} \mid \Lambda}(v)=S_{\{0\} \mid\{x<o\}}(v)=s(v),
$$

where we used Theorem 2 (twice) and the monotinicity of conditional entropy. Therefore, we obtain $s(v)=s(\mu)$, which by the variational principle implies that $v$ is an equilibrium state, and we obtain $S_{\{o\} \mid\{x<o\}}(\mu)=S_{\{\underline{o}\} \mid \Lambda}(\mu)$, which by Lemma 1 implies that

$$
\mu_{\{\underline{\{0\}}}\left(. \mid \sigma_{\{x<\underline{o}\}}\right)=\mu_{\{\underline{o}\}}\left(. \mid \sigma_{\Lambda}\right) .
$$

Lemma 2 provides the tool for deriving results in this section. To apply it, for an equilibrium state $\mu$, we have to supply a state $v$ with properties as listed in the lemma.

We now construct states $v$ to which we can apply Lemma 2. To this end, we present a new Markovization using a refinement of the method presented in the introductory section. A first step toward this new Markovization is in the next lemma, but first we introduce some special subsets of $\mathbb{Z}^{d}$. Denote the translation over one lattice spacing in the $k^{\text {th }}$ direction by $\tau_{k}$. Define

$$
\partial^{*} \Lambda \equiv \partial\left\{\Lambda^{c}\right\}
$$

for $\Lambda \subset \mathbb{Z}^{d}$.

For $k=d, \ldots, 1$, let $p_{k}$ be the "projection" $p_{k}: \mathbb{Z}^{d} \rightarrow \mathbb{Z}^{k}$ given by defining $p_{k} x \in \mathbb{Z}^{k}$ for $x \in \mathbb{Z}^{d}$ by

$$
p_{k} x=\left(x_{1}, \ldots, x_{k}\right)
$$

and define $p_{0} x=0 \in\{0\} \equiv \mathbb{Z}^{0}$.

In the following we use for $y \in \mathbb{Z}^{d}$ the notation [ ] as

$$
\left[p_{k} x<p_{k} y\right]=\left\{x \in \mathbb{Z}^{d} \mid p_{k} x<p_{k} y\right\}
$$

and

$$
\left[p_{k} y\right]=\left\{x \in \mathbb{Z}^{d} \mid p_{k} x=p_{k} y\right\} .
$$

Now, the set $\Delta_{y}^{k} \subset \mathbb{Z}^{d}$ is for $k=d, \ldots, 0$ and $y \in \mathbb{Z}^{d}$ defined as 


$$
\Delta_{y}^{k}=\partial^{*}\left[p_{k} x<p_{k} y\right] \cup\left[p_{k} y\right]
$$

Note that $\Delta_{y}^{0}=\mathbb{Z}^{d}$ and

$$
\Delta_{y}^{k-1}=\bigcup_{n=-\infty}^{+\infty} \tau_{k}^{n} \Delta_{y}^{k}
$$

for any $y \in \mathbb{Z}^{d}$ and $k=d, \ldots, 1$.

We also introduce $C^{k,+}$ and $C^{k,-}$ as

$$
C^{k,+}=\bigcup_{n=0}^{\infty} \tau_{k}^{n} \Delta_{o}^{k} \quad \text { and } \quad C^{k,-}=\bigcup_{n=-1}^{\infty} \tau_{k}^{n} \Delta_{\underline{o}}^{k} .
$$

Then

$$
C^{k,+}=\partial^{*}\left[p_{k} x<p_{k} \underline{o}\right] \cup p_{k}^{-1}\left\{\left(0, \ldots, 0, x_{k}\right), x_{k} \geqq 0\right\}
$$

and

$$
C^{k,-}=\partial^{*}\left[p_{k} x<p_{k} \underline{Q}\right] \cup p_{k}^{-1}\left\{\left(-1, \ldots,-1, x_{k}\right), x_{k} \leqq-2\right\} .
$$

Lemma 3. Take $k \in\{1, \ldots, d\}$. Let $v^{k}$ be a translation-invariant state on $\Omega_{\Delta_{o}^{k}}$. Then there exists a unique translation-invariant state $v^{k-1}$ on $\Omega_{\Delta_{o}^{k-1}}$ such that

1. $v_{\Delta_{o}^{k}}^{k-1}=v^{k}$,

2. $v_{\left[p_{k} o\right]}^{k-1}\left(. \mid \sigma_{C^{k,-}}\right)=v_{\left[p_{k} o\right]}^{k-1}\left(. \mid \sigma_{\partial^{*}\left[p_{k} x<p_{k} o\right]}\right)$.

Proof. Define $X^{n}=p_{k}^{-1}\left\{\left(0, \ldots, 0, x_{k}\right), x_{k}=n\right\}$ and put

$$
D^{n} \equiv \partial^{*}\left[p_{k} x<p_{k} \underline{0}\right] \cup\left\{X^{0} \cup \cdots \cup X^{n-1}\right\}=\bigcup_{m=0}^{n} \tau_{k}^{m}\left(\partial^{*}\left[p_{k} x<p_{k} \underline{0}\right]\right) .
$$

The state $v^{k}$ may be extended in a "Markovian" manner from $\Omega_{\Delta_{o}^{k}}$ to a measure $v^{+}$ on $\Omega_{C^{k,+}}$ by giving the projections $v^{+, n}$ of $v^{+}$on $\Omega_{D^{n}}$ for $n=0,1,2, \ldots$, as follows.

Define recursively, starting with $v^{+, 0} \equiv\left(v^{k}\right)_{\partial^{*}\left[p_{k} x<p_{k}\right]}$, the states $v^{+, n}$ by

1. $v_{D^{n-1}}^{+, n}=v^{+, n-1}$

2. $v_{X^{n-1}}^{+, n}\left(. \mid \sigma_{D^{n-1}}\right)=v_{X^{0}}^{k}\left(\tau_{k}^{-n} \cdot \mid \tau_{k}^{-n} \sigma_{\tau_{k}^{n} \partial^{*}\left[p_{k} x<p_{k} \underline{ }\right]}\right)$.

Informally the $n^{\text {th }}$ step in this recursion can be thought of as adding the spins in $X^{n-1}$ to those in $D^{n-1}$ using, as expressed by 2 . for each $n$ the same, up to translation, conditional probabilities to obtain the extension $v^{+, n}$ of the state $v^{+, n-1}$.

The extension $v^{+, 1}$ of $v^{+, 0}$ is $v^{k}$ and therefore translation-invariant. In particular $v_{\tau_{k} \partial^{*}\left[p_{k} x<p_{k} o\right]}^{+, 1}$ is equal, up to translation, to $v^{+, 0}$. But then it follows inductively that $v_{\tau_{k}^{n} \partial^{+}, n}^{\left.+, n p_{k} x<p_{k} o\right]}$ equals for each $n$, up to translation, $v^{+, 0}$ since for each $n$ the same conditional probabilities, up to translation, are used in 2 . Therefore $v^{+}$is translation invariant (and no technicalities involving sets of measure 0 can arise).

Take $v^{k-1}$ as the translation-invariant state on $\Omega_{\Delta_{o}^{k-1}}$ obtained from translation of $v^{+}$. It is obvious that this state $v^{k-1}$ is the unique state satisfying the conditions of the lemma.

Note that

$$
\Delta_{\underline{o}}^{1}=\partial^{*}\left[p_{1} x<p_{1} \underline{o}\right] \cup\left[p_{1} \underline{o}\right]=\left\{x_{1}=0,-1\right\},
$$


and thus when $k=1$, the state $v^{k-1}\left(=v^{0}\right)$ constructed in Lemma 3 from $v^{k}\left(=v^{1}\right)$ is precisely the Markov chain arising from $v^{k}$.

Lemma 4. Let $\mu$ be a translation-invariant state on $\Omega$. Then there exists a translation-invariant state $\bar{v}$ on $\Omega$ such that

1. $\bar{v}_{\Delta_{\underline{o}}^{d}}=\mu_{\Delta_{\underline{o}}^{d}}$,

2. $\bar{v}_{\{\underline{o}\}}\left(\cdot \mid \sigma_{\{x<o\}}\right)=\bar{v}_{\{\underline{o}\}}\left(\cdot \mid \sigma_{\partial^{*}\{x<o\}}\right)(\bar{v}$ almost surely).

Proof. Put $v^{d}=\mu_{\Delta_{o}^{d}}$ and construct, at each step using Lemma 3, the sequence of states $v^{k}$ on $\Omega_{\Delta_{o}^{k}}$ for $k=d-1, \ldots, 0$.

We will show that $\bar{v} \equiv v^{0}$ satisfies the condition of the lemma. The fact that $\bar{v}$ satisfies 1 . is evident and therefore we turn to proving 2. Property 2 . is the following Assertion for $m=d$.

Assertion (m).

$$
\bar{v}_{\left[p_{m} o\right]}\left(\cdot \mid \sigma_{\left[p_{m} x<p_{m}\right]}\right)=\bar{v}_{\left[p_{m} o\right]}\left(\cdot \mid \sigma_{\partial^{*}\left[p_{m} x<p_{m} o\right]}\right) .
$$

We will prove Assertion $(m)$ for $m=0, \ldots, d$ by induction on $m$. Assertion ( 0 ) is obviously true.

Take $m \in\{1, \ldots, d\}$ and assume that Assertion $(m-1)$ is true. Then in particular spins in

$$
Y_{\underline{o}}^{m-1} \equiv\left[p_{m} x<p_{m} \underline{o}\right] \backslash\left[p_{m-1} x<p_{m-1} \underline{o}\right]
$$

are independent of those in $\left[p_{m-1} x<p_{m-1} o\right]$ upon conditioning on the spins in $\partial^{*}\left[p_{m-1} x<p_{m-1} O\right]$, and therefore since

and

$$
\left[p_{m} x<p_{m} \underline{o}\right]=\left[p_{m-1} x<p_{m-1} \underline{o}\right] \cup Y_{\underline{o}}^{m-1}
$$

$$
C^{m,-}=\partial^{*}\left[p_{m-1} x<p_{m-1} o\right] \cup Y_{\underline{o}}^{m-1},
$$

we have from Assertion $(m-1)$ that

$$
\bar{v}_{\left[p_{m} \underline{0}\right]}\left(\cdot \mid \sigma_{\left[p_{m} x<p_{m}\right]}\right)=\bar{v}_{\left[p_{m} \underline{0}\right]}\left(\cdot \mid \sigma_{C^{m,-}}\right) .
$$

But by construction,

$$
\begin{aligned}
\bar{v}_{\left[p_{m} g\right]}\left(\cdot \mid \sigma_{C^{m,-}}\right) & =v_{\left[p_{m} o\right]}^{m-1}\left(\cdot \mid \sigma_{C^{m,-}}\right)=v_{\left[p_{m} o\right]}^{m-1}\left(\cdot \mid \sigma_{\partial^{*}\left[p_{m} x<p_{m}\right]}\right) \\
& =\bar{v}_{\left[p_{m}\right]}\left(\cdot \mid \sigma_{\partial^{*}\left[p_{m} x<p_{m}\right]}\right),
\end{aligned}
$$

which finishes the proof of Assertion $(m)$.

Since Assertion $(d)$ is property 2. This completes the proof of this lemma.

Theorem 3. Any translation-invariant Gibbs state $\mu$ for the potential $\Phi$ has the Markov property

Hence

$$
\mu_{\{\underline{o g}\}}\left(\cdot \mid \sigma_{\{x<\underline{0}\}}\right)=\mu_{\{\underline{o g}\}}\left(\cdot \mid \sigma_{\partial^{*}\{x<\underline{0}\}}\right) .
$$

$$
s(\mu)=S_{\{\underline{\{0}\} \mid \partial^{*}\{x<\underline{0}\}}(\mu) .
$$

Proof. Let $\bar{v}$ be the state associated with $\mu$ as in Lemma 4. The theorem follows from application of Lemma 2 with $v=\bar{v}$ and $\Lambda=\partial^{*}\{x<\underline{o}\}$. 
Theorem 0 is essentially an immediate consequence of Lemma 2:

Corollary 1. Let $\bar{\mu}$ be the Markovization of an equilibrium state $\mu$ as in the introductory section. Then $\bar{\mu}$ is an equilibrium state.

Proof. Set $\Lambda=\left\{\{x<\underline{o}\} \cap\left\{x_{1}=0\right\}\right\} \cup\left\{x_{1}=-1\right\}$ and apply Lemma 2 with $v=\bar{\mu}$. To see that condition (b) of Lemma 2 is met for this choice for $\Lambda$ and $v$, note that, upon conditioning on the spins in $\left\{x_{1}=-1\right\}$, the spins in $\left\{x_{1}=0\right\}$ become independent of those in $\left\{x_{1} \leqq-2\right\}$ by the global Markov property for $\left\{x_{1} \geqq 0\right\}$ of $\bar{\mu}$.

The following example shows how failure of the condition $C(\mu)$ leads to failure of the global Markov property for $\Lambda_{+}$for certain equilibrium states. Thus this example illustrates the second assertion of Theorem 0 .

Example. Let $M=\{-1,+1\}^{\mathbb{Z}}$ and for $m=\left\{m_{k}\right) \in M$ let $\mu_{m}$ be the state on $\{-1,+1\}^{\mathbb{Z}^{3}}$ for which the layers $\left\{x_{1}=k\right\}$ are independent and $\left(\mu_{m}\right)_{\left\{x_{1}=k\right\}}$ is the $m_{k}$-phase of the two-dimensional nearest-neighbour Ising model. Then $\mu_{m}$ is a Gibbs state for the potential $\Phi$, which in $(y-z)$ planes is the interaction of the two-dimensional Ising model and which gives no interaction between different $(y-z)$ planes. Thus we have a collection of independent two-dimensional Ising models. We assume that the Ising model is in the two-phase regime in which the $(+1)$ phase differs from the $(-1)$ phase. For $\rho$ a state on $M$ we define the state $\mu_{\rho}$ on $\{-1,+1\}^{\mathbb{Z}^{3}}$ by

$$
\mu_{\rho}=\int_{M} \rho(d m) \mu_{m}
$$

Then $\mu_{\rho}$ is a Gibbs state for $\Phi$ and (3) is its decomposition into extremal Gibbs states. Furthermore, $\mu_{\rho}$ is $\mathbb{Z}^{d}$-ergodic if $\rho$ is $\mathbb{Z}$-ergodic. Now let $\rho$ be a non-Markovian state on $M$. Then $\mu_{\rho}$ does not satisfy the global Markov property for $\Lambda_{+} \subset \mathbb{Z}^{3}$. In particular, if besides being non-Markovian, the state $\rho$ is $\mathbb{Z}$-ergodic, then $\mu_{\rho}$ is an extremal translation-invariant Gibbs state which does not have the global Markov property for $\Lambda_{+}$.

The following consequence of Theorem 3 might be of interest when $d=2$.

Corollary 2. For any pair of translation-invariant Gibbs states $\mu$ and $v$ for $\Phi$, we have

$$
\mu_{\Delta_{o}^{d}}=v_{\Delta_{o}^{d}} \Leftrightarrow \mu_{\Delta_{o}^{d-1}}=v_{\Delta_{o}^{d}-1}
$$

Proof. Immediate from Theorem 3.

Theorem 3 also enables one to formulate a "minimal" variational principle [6], which is stated in terms of states on essentially $(d-1)$-dimensional systems, and which enables the free energy $f_{\Phi}$ to be computed.

Theorem 4. Let $v$ be a translation-invariant state on $\Omega_{\Delta_{o}^{d}}$. Set $S_{\partial}(v)=S_{\{\underline{\underline{o}}\} \mid 0^{*}\{x<\underline{0}\}}(v)$. Then

(a) $v\left(e_{\Phi}\right)-s_{\partial}(v) \geqq f_{\Phi}$,

(b) $v\left(e_{\Phi}\right)-s_{\partial}(v)=f_{\Phi}$ if, and only if, there is a translation-invariant Gibbs state $\tilde{v}$ on $\Omega$ with $\tilde{v}_{\Delta_{o}^{d}}=v$. 
In particular

$$
f_{\Phi}=\inf _{v}\left[v\left(e_{\Phi}\right)-s_{\partial}(v)\right]
$$

where the infimum is taken over the set of translation-invariant states on $\Omega_{\Delta_{o}^{d}}$.

Proof. Let $\tilde{v}$ be a translation-invariant state on $\Omega$ such that

1. $\tilde{v}_{\Delta_{o}^{d}}=v$,

2. $\tilde{v}_{\{\underline{o}\}}\left(\cdot \mid \sigma_{\{x<o\}}\right)=\tilde{v}_{\{\underline{o}\}}\left(\cdot \mid \sigma_{\hat{o}^{*}\{x<o\}}\right) \quad(\tilde{v}$ almost surely).

Such a state exists by the construction presented in the proof of Theorem 3 . Then

$$
v\left(e_{\Phi}\right)-s_{\partial}(v)=\tilde{v}\left(e_{\Phi}\right)-s_{\partial}\left(\tilde{v}_{\Delta_{o}^{d}}\right)=\tilde{v}\left(e_{\Phi}\right)-s(\tilde{v}) \geqq f_{\Phi},
$$

and equality holds if, and only if, $\tilde{v}$ is a Gibbs state, proving (a) and the only if part of (b). If $\tilde{v}$ is a translation-invariant Gibbs state with $\tilde{v}_{\Delta_{o}^{d}}=v$, then

$$
s(\tilde{v})=s_{\partial}(\tilde{v})=s_{\partial}(v),
$$

and it follows that

$$
v\left(e_{\Phi}\right)-s_{\partial}(v)=\tilde{v}\left(e_{\Phi}\right)-s(\tilde{v})=f_{\Phi} .
$$

As yet another consequence of Theorem 3 we obtain Theorem 5:

Theorem 5. Let $\mu$ be a translation-invariant Gibbs state for $\Phi$ and consider the condition $C(\mu)$, which was introduced in Theorem 0 . If $C(\mu)$ holds, then $\mu$ has the global Markov property for $\{x<\underline{o}\}^{c}$.

Proof. Immediate from Theorem 0 and Theorem 3.

We will now extend part of the results obtained thus far for translation-invariant Gibbs states to periodic Gibbs states. We first need a technical lemma. When $\mu$ is a state, we denote the set of functions in $L^{1}(\mu)$ that are measurable with respect to $B_{\Lambda}, \Lambda \subset \mathbb{Z}^{d}$ by $L^{1}\left(\mu ; B_{\Lambda}\right)$.

Lemma 5. Let $\tau$ be a translation and let $\tilde{\Lambda}$ be a subset of $\mathbb{Z}^{d}$ such that for all finite $\Lambda \subset \mathbb{Z}^{d}, a k \in \mathbb{Z}$ exists such that $\tau^{k} \Lambda \subset \tilde{\Lambda}$. Then, if $\mu$ is a $\tau$-invariant state, for all $f \in L^{1}(\mu)$ which are $\tau$-invariant, i.e., $\tau f=f$, we have $f \in L^{1}\left(v ; B_{\tilde{\Lambda}}\right)$.

Proof. This is an immediate consequence of $\tau$-invariance of $\mu$ and the fact that $\bigcup_{\Lambda \text { finite }} L^{1}\left(\mu, B_{\Lambda}\right)$ is dense in $L^{1}(\mu)$.

Lemma 6. Let $\tau$ be a translation for which, for each finite $\Lambda \subset \mathbb{Z}^{d}, k \in \mathbb{Z}$ exists such that $\tau^{k} \Lambda \subset\{x<\underline{o}\}$. Let $\mu$ be a translation-invariant Gibbs state and let $v$ be $a$ $\tau$-invariant state. If $v \ll \mu$ ( $v$ is absolutely continuous with respect to $\mu$ ), then

$$
v_{\{\underline{o}\}}\left(\cdot \mid \sigma_{\{x<\underline{o}\}}\right)=v_{\{\underline{o}\}}\left(\cdot \mid \sigma_{\partial *\{x<o\}}\right) .
$$

Proof. Note that $(d v / d \mu) \in L^{1}(\mu)$ is $\tau$-invariant. So $(d v / d \mu) \in L^{1}\left(\mu ; B_{\{x<0\}}\right)$ by the previous lemma. Let $E_{\omega}(f \mid \Lambda)$ be the conditional expectation for a state $\omega$ of a function $f$ with respect to $B_{\Lambda}$. Since $(d v / d \mu) \in L^{1}\left(\mu ; B_{\{x<\underline{o}\}}\right)$, we have

$$
E_{v}(f \mid\{x<\underline{o}\})=E_{\mu}(f \mid\{x<\underline{o}\}) .
$$


If $f$ is a function that depends only on the spin at $\underline{o}$ we have, by Theorem 3 ,

$$
E_{\mu}(f \mid\{x<\underline{o}\})=E_{\mu}\left(f \mid \partial^{*}\{x<\underline{o}\}\right),
$$

and it follows that the same property holds for the state $v$. This proves the lemma.

Theorem 6. Let $v$ be a periodic Gibbs state for $\Phi$, i.e. $v$ is a Gibbs state that is invariant under a subgroup, $G$, of finite index in $\mathbb{Z}^{d},\left|\mathbb{Z}^{d} / G\right|<\infty$. Then

$$
v_{\{\underline{0}\}}\left(\cdot \mid \sigma_{\{x<0\}}\right)=v_{\{0\}}\left(\cdot \mid \sigma_{\partial^{*}\{x<0\}}\right)(v \text { almost surely). }
$$

Proof. There exist $N \in \mathbb{N}$ and translations $\left\{\tau^{(n)}\right\}_{n=1, \ldots, N}$ such that

$$
\mu \equiv \frac{1}{N+1}\left[v+\sum_{n=1}^{N} \tau^{(n)} v\right]
$$

is a translation-invariant Gibbs state. By construction $v \ll \mu$, and by choosing $\tau=\tau_{1}^{k}$ for some $k \in \mathbb{Z}$, we can invoke the previous lemma to complete the proof.

\section{Markov Properties and Invariance}

In the previous section we deduced Markov properties for invariant states. It is interesting to note that, conversely, knowledge of Markov properties can be used to "enhance" invariances of Gibbs states. We need a few extra assumptions however. First, we assume that the potential $\Phi$, beside being translation-invariant, is also $\theta_{0}$-invariant, where $\theta_{0}$ is reflection in the layer $\left\{x_{1}=0\right\}$.

Furthermore, we consider condition $C_{\Phi}$ :

$$
\begin{array}{ll}
C_{\Phi} \quad \text { Every translation- and } \theta_{0} \text {-invariant Gibbs state for } \Phi \\
\text { satisfies the global Markov property for }\left\{x_{1}>0\right\} .
\end{array}
$$

Remark. By arguments analogous to those that led to Theorem 6, condition $C_{\Phi}$ is equivalent to the same condition with "periodic state" substituted for "translation-invariant state." Let $v, \mu$ be two states on $\Omega$ with $v \ll \mu$ and let $\mu$ be $\theta_{0}$-invariant. If $d v / d \mu$ is measurable with respect to $B_{\left\{x_{1}=0\right\}}$, then $d v / d \mu$ is $\theta_{0}$-invariant and hence $v$ is also $\theta_{0}$-invariant.

Theorem 7, cf. [8]. Let $G$ be a group of finite index and let $\mu$ be a Gibbs state invariant under $G$. If $C_{\Phi}$ holds, then $\mu$ is invariant under $\theta_{k}$, where $\theta_{k}$ is reflection in the layer $\left\{x_{1}=k\right\}$, for all $k \in \mathbb{Z}$. In particular $\mu$ is invariant under $\tau_{1}^{2}$.

Proof. There exist $N \in \mathbb{N}$ and translations $\left\{\tau^{(n)}\right\}_{n=1, \ldots, N}$ such that

$$
\mu^{\prime} \equiv \frac{1}{N+1}\left(\mu+\sum_{n=1}^{N} \tau^{(n)} \mu\right)
$$

is translation-invariant.

Let $\tilde{\mu}=\frac{1}{2}\left(\mu^{\prime}+\theta_{0} \mu^{\prime}\right)$. Then $\tilde{\mu}$ is a translation- and $\theta_{0}$-invariant Gibbs state and $\mu \ll \tilde{\mu}$. Since $(d \mu / d \tilde{\mu}) \in L^{1}(\tilde{\mu})$ is G-invariant, it follows by applying Lemma 5 (twice) that $(d \mu / d \tilde{\mu}) \in L^{1}\left(\mu ; B_{\left\{x_{1} \leqq 0\right\}}\right)$ and $(d \mu / d \tilde{\mu}) \in L^{1}\left(\tilde{\mu} ; B_{\left\{x_{1} \geqq 0\right\}}\right)$.

Hence, with conditional expectations for the state $\tilde{\mu}$, 


$$
\frac{d \mu}{d \tilde{\mu}}=E\left(\frac{d \mu}{d \tilde{\mu}} \mid\left\{x_{1}<0\right\}^{c}\right)=E\left(\frac{d \mu}{d \tilde{\mu}} \mid\left\{x_{1}>0\right\}^{c}\right)=E\left(\frac{d \mu}{d \tilde{\mu}} \mid\left\{x_{1}=0\right\}\right),
$$

where in the third equality we used that $\tilde{\mu}$ has the global Markov property for $\left\{x_{1}>0\right\}$ by condition $C_{\Phi}$. Therefore, $\mathrm{d} \mu / \mathrm{d} \tilde{\mu}$ is measurable with respect to $B_{\left\{x_{1}=0\right\}}$, and it follows from remarks made just prior to this theorem that $\mu$ is $\theta_{0}$-invariant. But $\tilde{\mu}$ is also $\theta_{k}$-invariant and analogous arguments therefore give that $\mu$ is $\theta_{k}$-invariant for any $k \in \mathbb{Z}$.

Note that Theorem 7 implies that $C_{\Phi}$ is in fact equivalent to $C_{\Phi}^{\prime}$ where

$$
\begin{aligned}
& C_{\Phi}^{\prime} \quad \text { Every translation-invariant state for } \Phi \text { satisfies } \\
& \text { the global Markov property for }\left\{x_{1}>0\right\}
\end{aligned}
$$

Let $v$ be a Gibbs state. Then $v$ has an integral representation, or decomposition,

$$
v=\int m_{v}\left(d v^{\prime}\right) v^{\prime}
$$

where $m_{v}$ is a measure on the space of states on $\Omega$ which is the unique measure that is concentrated on the set of extremal states. The decomposition (5) is the decomposition of $v$ into extremal Gibbs states. Consider condition $\bar{C}_{\Phi^{\prime}}$

$$
\begin{aligned}
& \bar{C}_{\Phi} \quad \text { Every translation-invariant Gibbs state for } \Phi \text { has } \\
& \text { the global Markov properties for }\left\{x_{i}>0\right\}, i=1, \ldots, d
\end{aligned}
$$

Let $\theta_{0}^{(k)}$ be reflection in the layer $\left\{x_{k}=0\right\}$. Assume for the next corollary that $\Phi$ is $\theta_{0}^{(k)}$-invariant. Note that with this condition on $\Phi$ as well as condition $\bar{C}_{\Phi}$ every periodic Gibbs state is $\mathbb{Z}_{e v}^{d}$-invariant (recall $\mathbb{Z}_{e v}=\{n \in \mathbb{Z} \mid n$ is even $\}$ ).

Corollary 3. Let $v$ be a $\mathbb{Z}_{\text {ev }}^{d}$-ergodic Gibbs state for $\Phi$ and let $v=\int m_{v}\left(d v^{\prime}\right) v^{\prime}$ be its decomposition into extremal Gibbs states. If $\bar{C}_{\boldsymbol{\Phi}}$ holds then exactly one of the following two statements is true:

1. $m_{v}$ is the Dirac measure at $v$, i.e., $v$ is an extremal Gibbs state,

2. $m_{v}$ is purely nonatomic.

If (2) is true, then we have in particular that there are uncountably many extremal Gibbs states.

Proof. The only nonevident assertion in the Corollary is the statement that nonextremality of $v$ implies that $m_{v}$ is purely nonatomic. Therefore, assume that $v$ is not an extremal Gibbs state. First, we observe that the measure $m_{v}$ is $\mathbb{Z}_{e v}^{d}$-invariant and is, moreover, ergodic for $\mathbb{Z}_{e v}^{d}$. Now let $\mu_{o}$ be an arbitrary state on $\Omega$ and consider $S_{o}=\left\{\tau \mu_{o}\right\}_{\tau \in \mathbb{Z}_{e v}^{d}}$. Clearly $S_{o}$ is $\mathbb{Z}_{e v}^{d}$-invariant. Thus, by ergodicity of $m_{v}$, one of two situations may arise,

(a) $m_{v}\left(S_{o}\right)=0$

(b) $m_{v}\left(S_{o}\right)=1$.

Assume, ad absurdum, that (b) holds. Since, by $\mathbb{Z}_{e v}^{d}$-invariance of $m_{v}$, each element in $S_{o}$ has equal weight, the set $S_{o}$ has to be finite and thus $m_{v}$ is concentrated on a finite set. Hence 


$$
v=\frac{1}{\left|S_{o}\right|} \sum_{v^{\prime} \in S_{o}} v^{\prime},
$$

where the states in $S_{o}$ are mutually disjoint and extremal Gibbs states. But such a representation of $v$ into extremal Gibbs states implies by its uniqueness that each of the states in $S_{o}$ is invariant under some subgroup of finite index. By Theorem 7, applied "in each lattice direction," it follows that each element in $S_{o}$ is $\mathbb{Z}_{e v}^{d}$-invariant. The $\mathbb{Z}_{e v}^{d}$-ergodicity of $v$ implies $\left|S_{o}\right|=1$. Hence $v$ is extremal Gibbs contradicting the assumption that $v$ is not an extremal Gibbs state. Therefore (b) is false and (a) holds, i.e., $m_{v}\left(S_{o}\right)=0$. In particular $m_{v}\left(\left\{\mu_{o}\right\}\right)=0$. As $\mu_{o}$ was arbitrary, this proves that $m_{v}$ is purely nonatomic.

\section{Concluding Remarks}

Sections 3 and 4 contain the main results of this paper. These results, however, can easily be generalised in several directions as we indicate briefly below:

1. Define $L_{+} \subset \mathbb{Z}^{d}$ by

$$
\left.L_{+}=\left\{0, \ldots, 0, x_{d}\right), x_{d} \geqq 0\right\} .
$$

By using Theorem 3, we can easily deduce that for each translation-invariant Gibbs state $\mu$,

$$
\mu_{L_{+}}\left(\cdot \mid \sigma_{\{x<\underline{0}\}}\right)=\mu_{L_{+}}\left(\cdot \mid \sigma_{\partial^{*}\{x<\underline{o}\}}\right),
$$

which is a strengthening of Theorem 3 .

2. By blocking spins we can reduce any finite-range potential to an interaction-round-faces potential. In this way we can obtain results for finite-range potentials analogous to the ones presented here.

3. Also, by using a spin-blocking technique, we can generalise the entropy formula of Theorem 3 to include cases where the state $\mu$ is periodic.

4. The result that

$$
\mu_{L_{+}}\left(\cdot \mid \sigma_{\{x<\underline{o}\}}\right)=\mu_{+}^{L}\left(\left.\cdot\right|_{\partial^{*}\{x<\underline{o}\}}\right),
$$

for any equilibrium state $\mu$ (see the first remark in this section) can be generalised to more general volumes, or subsets, in $\mathbb{Z}^{d}$. Rather than giving a description for general $d$ we describe the generalisation we have in mind for $d=2$. Let $\alpha: \mathbb{Z} \rightarrow \mathbb{Z} \cup\{ \pm \infty\}$ be a function, such that

$$
\alpha(y)=\left\{\begin{array}{l}
+\infty \text { for } y \leqq y_{\min } \in \mathbb{Z} \\
\text { finite for } y_{\min }<y<y_{\max } \in \mathbb{Z}, \\
-\infty \text { for } y \geqq y_{\max }
\end{array}\right.
$$

where $y_{\min }$ and $y_{\max }$ are in $\mathbb{Z}$, i.e., the set of points in $\mathbb{Z}$ where $\alpha$ is finite is an interval (in $\mathbb{Z}$ ). Define $V_{\alpha} \subset \mathbb{Z}^{2}$ by $V_{\alpha}=\left\{\alpha\left(x_{1}\right) \geqq x_{2}\right\}$ and set $V_{+}=\left\{\alpha\left(x_{1}\right)\right.$ finite, $\left.\alpha\left(x_{1}\right)<x_{2}\right\}$. Then for each translation-invariant Gibbs state $\mu$, we have

$$
\mu_{V_{+}}\left(\cdot \mid \sigma_{V_{\alpha}}\right)=\mu_{V_{+}}\left(\cdot \mid \sigma_{\partial^{*} V_{\alpha}}\right) \text {. }
$$

The proof of this involves steps analogous to those taken to prove Theorem 3 . 
However, not all of the arguments and constructions can now be done within the set of translation-invariant states. Instead periodic states are used. The details are left to the reader.

5. We have assumed that $\Omega_{o}$ is a finite set, but all results also hold when $\Omega_{o}$ is a compact metric space.

\section{References}

1. Fölmer, H.: On the global Markov property; Quantum fields -, algebras, processes. Streit, L. (ed.), pp. 293-302. Wien, New York: Springer 1980

2. Goldstein, S.: Remarks on the global Markov property. Commun. Math. Phys. 74, 223-234(1980)

3. Bellissard, J., Hoegh-Krohn, R.: Compactness and the maximal Gibbs state for random Gibbs fields on a lattice. Commun. Math. Phys. 84, 297-327 (1982)

4. Israel, R. B.: Some examples concerning the global Markov property. Commun. Math. Phys. 105, 669-673 (1986)

5. Kuik, R.: The global Markov property for equilibrium states which are determined by correlations in a strip. Commun. Math. Phys. 115, 177-189 (1988)

6. Schlijper, A. G.: On some variational approximations in two-dimensional classical lattice systems. J. Stat. Phys. 40, 1-27 (1985)

7. Katznelson, Y., Weis, B.: Commuting measure-preserving transformations. Israel J. Math. 12, 161-173 (1972)

8. Kuik, R.: Markov and stability properties of equilibrium states for nearest-neighbor interactions. Commun. Math. Phys. 115, 529-552 (1988)

9. Ruelle, D.: Thermodynamic formalism. Reading, MA: Addison Wesley 1978

Communicated by M. Aizenman

Received February 13, 1989; in revised form June 14, 1989 